\title{
Innovación en la enseñanza de posgrado en comunicación: el aprendizaje-servicio como estrategia pedagógica.
}

\author{
Alejandro Álvarez NoBell \\ Universidad Nacional de Córdoba (Argentina) \\ aalvareznobell@gmail.com \\ Nerea VAdillo BengoA \\ Universidad San Jorge (España) \\ nvadillo@usj.es
}

\begin{abstract}
Resumen
Las propuestas de aprendizaje servicio -APS- aplicado al posgrado en comunicación, quieren dotar de más significado social y prácticos a los saberes académicos y formar también en la responsabilidad social. El presente artículo describe la práctica formativa de Aprendizaje-Servicio llamada "Maratón Solidario en Comunicación Corporativa: Protagonistas del Cambio" que de manera consecutiva, en los años 2012 y 2013 ha tenido lugar en Zaragoza, promovida por el BrandPR Máster Universitario en Marketing y Comunicación Corporativa de la Universidad San Jorge.
\end{abstract}

Palabras clave: Comunicación; Aprendizaje Servicio; ApS; Innovación Docente; Responsabilidad Social Universitaria; Posgrado

Innovation in postgraduate education in communication: service learning as a pedagogical strategy.

\begin{abstract}
The service-learning proposals APS-applied to graduate in communication, they want to provide more social and practical meaning to academic knowledge and also form social responsibility. This article describes the practice of service-learning training called "Solidarity Marathon Corporate Communication: Protagonists of Change" that in a row, in 2012 and 2013 was held in Zaragoza, sponsored by BrandPR Master in Marketing and Communication Corporate San Jorge University.
\end{abstract}

Keywords: Communication, Learning Service; ApS, Teaching Innovation, Social Responsibility University, Postgraduate

\section{Referencia normalizada:}

Álvarez Nobell, A. y Vadillo Bengoa, N. (2013) Innovación en la enseñanza de posgrado en comunicación: el aprendizaje-servicio como estrategia pedagógica. Historia y Comunicación Social. Vol. 18. $\mathrm{N}^{\circ}$ Especial Diciembre. Págs. 263-277.

Sumario: 1. Introducción. 2. Metodología. 3. Marco Teórico. 3.1. Aprendizaje Servicio. 4. Desarrollo: Maratón Solidario de Comunicación Corporativa. 4.1. Escenarios 4.2. El diseño de la práctica de aprendizaje servicio. 4.3. La experiencia. 5. Discusiones y Conclusiones finales. 6. Referencias Bibliográficas. 


\section{Introducción}

Hoy la educación debe orientar sus esfuerzos en fomentar prácticas que permitan a las generaciones más jóvenes participar activa y responsablemente en la construcción de su realidad. La Universidad requiere la aplicación de prácticas innovadoras, coherentes con estas nuevas necesidades y contextos. Así, surgen diversas propuestas pedagógicas que desde una perspectiva participativa y solidaria buscan dotar al ámbito formativo de un significado social y formar en la responsabilidad.

Es el caso del Aprendizaje Servicio -APS-, una estrategia metodológica innovadora que tiene por finalidad favorecer durante el proceso formativo, la implicancia y el compromiso de los estudiantes con la comunidad y el entorno, mediante la incorporación del servicio voluntario en el aula (Martínez-Odría, 2007). El concepto de Aprendizaje Servicio o Service Learning surge en Estados Unidos en 1969. En las bases de esta metodología se encuentran: los trabajos pioneros de Jane Addams en 1904 (Daynes y Longo, 2004: 5), el aprendizaje experiencial del pedagogo norteamericano John Dewey, y las corrientes del constructivismo social. Le siguieron acciones en las Universidades Latinoamericanas de México, Costa Rica, y Colombia (Cecchi, 2006: 11). Así se fue abriendo camino globalmente el uso de esta innovadora metodología de enseñanza.

Puig Rovira (2009: 9) lo define como:

Una metodología de alto poder formativo que combina en una sola actividad el aprendizaje de contenidos, competencias y valores, con la realización de tareas de servicio a la comunidad. El conocimiento se utiliza para mejorar algo en la comunidad y el servicio se convierte en una experiencia de aprendizaje que proporciona conocimientos y valores

El fondo de este planteamiento reside en la idea de establecer nuevos espacios de interacción entre la Universidad y la comunidad; conjuntamente con el involucramiento de otros agentes sociales en el proceso educativo de los alumnos, utilizando el servicio voluntario como herramienta para conseguirlo (Martínez, 2010). Las universidades son cada vez más conscientes de que su función no se puede realizar con calidad al margen de la responsabilidad social que como instituciones se les exige. Y no solo por ello sino porque también la formación de los estudiantes que acogen les debe preparar para ejercer como profesionales en un contexto social, tecnológico y cultural cada vez más complejo (Martínez, 2010).

En ese sentido, las propuestas de aprendizaje servicio -APS- en el ámbito universitario quieren dotar de más significado social a los aprendizajes académicos y formar en la responsabilidad social. Y lo hacen mediante prácticas de voluntariado que surgen de propuestas docentes que enfatizan enfoques orientados con el objetivo de que el estudiante se implique y se comprometa más con la comunidad y en el ejercicio de responsabilidad ética que, desde su futura profesión, tendrá que ejercer.

Así, se describe a continuación, mediante un diseño metodológico de observación participante, la práctica formativa de Aprendizaje-Servicio y Responsabilidad Social 
Universitaria llamada "Maratón Solidario en Comunicación Corporativa: Protagonistas del Cambio" que de manera consecutiva, en los años 2012 y 2013 ha tenido lugar en Zaragoza, promovida por el Brand PR Máster Universitario en Marketing y Comunicación Corporativa de la Universidad San Jorge.

En particular, la práctica consistió en una jornada de todo un día, con sesiones paralelas de dos horas cada una, en las que gratuitamente un conjunto de organizaciones del tercer sector fueron asesoradas para alcanzar soluciones efectivas a sus problemas actuales de comunicación. De la sesión participan integrantes de la ONG, el alumno tutor y un panel de tres profesionales externos expertos en comunicación convocados ad-hoc. Primeramente, los estudiantes participantes como tutores fueron capacitados con el objetivo de conocer de cerca y en profundidad el tercer sector en España, para luego tomar contacto con una de las organizaciones que aplicaron a la convocatoria y fueron seleccionadas. Ya en la sesión, el propio día del Maratón, los estudiantes presentaron a la organización, a sus integrantes asistentes, el diagnóstico realizado previamente; para después, con el aporte de un panel de consultores, desarrollar propuestas de soluciones concretas, que finalmente se presentarían como recomendaciones en un informe escrito. La extensa jornada (10 a 20 horas) culmina con una conferencia integradora de la experiencia "Claves para la comunicación solidaria en la actualidad", destinada a participantes, estudiantes del grado, ONG's y público en general.

Sin duda que la iniciativa permitió demostrar que la formación que se imparte también puede aportar valor y contribuir con la misión y gestión de las organizaciones solidarias. Pero fundamentalmente, que la Universidad puede y debe facilitar los espacios que permitan vincular a profesionales, profesores, estudiantes, organizaciones y empresas para trabajar conjuntamente en la construcción de una sociedad más justa y solidaria.

\section{Metodología}

El diseño metodológico aplicado empleo una metodología cualitativa de observaciones participantes. Sobre el desarrollo de la práctica, ambos, como investigadores tuvimos posibilidad de formar parte del diseño de la estrategia y de la implementación. Como dispositivo se emplearon tablas para el registro de la observación, complementado con registro y análisis documental. El caso seleccionado corresponde con programa oficial de posgrado de la Facultad de Comunicación, que desde 2006 la Universidad privada San Jorge, en Zaragoza (España) lleva adelante impartiendo bajo el nombre de BrandP Máster Universitario en Marketing y Comunicación Coporativa. 


\section{Marco teórico}

\subsection{Aprendizaje-servicio}

Cuando el estudiante aprende a través de una experiencia de servicio organizado que impliquen conocimiento (ligados al currículo académico) y ayuden a su vez a la sociedad, estamos antes una práctica de Aprendizaje Servicio o ApS (Naval 2010). Así, diremos que el Aprendizaje-Servicio es según Puig y Palos (2006: 61):

una propuesta educativa que combina procesos de aprendizaje y de servicio a la comunidad en un solo proyecto bien articulado en el que los participantes se forman al trabajar sobre necesidades reales del entorno con el objetivo de mejorarlo.

Esta propuesta pedagógica permite vincular contenidos curriculares y competencias trasversales (Martínez y Francisco, 2005) como el trabajo en equipo, toma de decisiones, liderazgo, entre otras; facilitando la relación entre experiencia y conocimiento. Sin embargo, no toda actividad práctica se corresponde con ApS, ni tampoco son voluntariados. Según Araújo y Arantes (2010) debe mediar interacción entre el sujeto que aprende y los objetos del conocimiento, y es fundamental que el estudiante asuma el protagonismo en la acción educativa, mediado y orientado por la sociedad y sus agentes. Por eso, se el aprendizaje se construye sobre las necesidades reales del entorno (Martínez 2010).

Para Puig y Palos (2006) las prácticas de ApS son un método apropiado para la educación formal y no formal, válido para todas las edades y aplicable en distintos espacios temporales. A su vez, proponen llevar a cabo un servicio auténtico a la comunidad que permita aprender y colaborar en un marco de reciprocidad; al tiempo que desencadenan procesos sistemáticos y ocasionales de adquisición de conocimientos y competencias para la vida. Significan una pedagogía de la experiencia y la reflexión que precisan de una red de alianzas entre las instituciones educativas y las entidades sociales que facilitan servicios a la comunidad; y lograr de ese modo provocar efectos en el desarrollo personal, cambios en las instituciones educativas y sociales que lo impulsan, y mejoras en el entorno comunitario que recibe el servicio.

En la actualidad es The National Service-Learning Clearinghouse (NSLC) la fuente americana más completa de Service-Learning. En España, dicen Marta Lazo y Aldea (2012), no se ha producido una verdadera aproximación y un desarrollo de estos proyectos con carácter más generalizado (Martínez-Odría, 2007; Puig y Palos, 2007; Martínez (ed.), 2008; Puig (coord.) 2009; Francisco y Moliner, 2010; Folgueiras, Luna y Puig, 2013) hasta la última década. 


\section{Desarrollo: maratón solidario de comunicación corporativa}

\subsection{Escenarios}

En el marco de la Facultad de Comunicación y de una oferta de posgrado, la posibilidad de diseñar una estrategia de innovación docente sin duda se presenta atravesada por un contexto que determina experiencia. En este caso, en las dos ediciones, nos encontramos que existía una la creciente necesidad de llevar adelante acciones de comunicación por parte de las ONG's que permitiesen mejorar sus programas de acción, fortalecer y profesionalizar sus actividades. Por lo general, éstas se ven dificultadas por la falta de conocimiento del aporte que puede brindar la comunicación a ello, dando como consecuencia un escaso desarrollo de estas posiciones y áreas (de marketing social y comunicación) en el tercer sector. Esto significaba dotar al tercer sector de un gran potencial para la generación de puestos de trabajo en el campo de la comunicación y el marketing motivado por el gran desarrollo y auge de estas organizaciones demandadas por la situación socioeconómica actual.

Por otro lado, había un deber de que la Universidad se constituyese como un espacio de integración y vínculo entre profesionales experimentados, estudiantes en formación y la realidad social, favoreciendo el compromiso y la responsabilidad; al tiempo que pusiera en práctica estratégicas que representasen de alto valor pedagógico para los estudiantes, en tanto significarán una posibilidad de experimentar en casos reales y bajo la supervisión de expertos profesionales en la materia, la puesta en práctica de habilidades y competencias adquiridas durante el Programa de Máster.

Desde la faz interna de la institución Universidad, existía una necesidad de articular experiencias que favorezcan el voluntariado y resulten de la sinergia de distintas ámbitos de la Universidad: Estudiantes, Información Universitaria Posgrados, Instituto de Humanismo y Sociedad, Unidad de Orientación Profesional y Empleo, Profesores de la Facultad de Comunicación, Personal Técnico y de Gestión y equipo del Máster.

Finalmente existían experiencias de éxito anteriores realizadas en Argentina desde el 2006 organizada por el Consejo Profesional de Relaciones Públicas de la República Argentina ${ }^{1}$, lo cual posibilito contar con una organización internacional asesora en la implementación de esta iniciativa pionera en Europa y España. También fue clave contar con la colaboración, verificación y asesoramiento por parte de Cáritas Diocesana de Zaragoza ${ }^{2}$, como referente del tercer sector y de la Asociación de Directores

1 [Disponible en http://www.rrpp.org.ar/index.php?page=Novedades. Novedades\&id=136\&pageindex $=0 \&$ seccion $=]$ Consultado el 10 de junio de 2013.

2 [Disponible en http://bibliotecacaritaszgz.blogspot.com.es/2012/04/ caritas-colabora-con-la-usj-enuna.html] Consultado el 10 de junio de 2013. 
de Comunicación (DIRCOM) con sede en Aragón ${ }^{3}$, como máximo representantes de expertos en la materia.

4.2 El diseño de la práctica de aprendizaje servicio

La práctica del Maratón Solidario de Comunicación Corporativa tuvo como objetivos propuestos:

Aplicar en casos reales las competencias y habilidades profesionales adquiridas durante el Programa de Máster.

Que los estudiantes capaciten y brinden asesoramiento gratuito a organizaciones de la sociedad civil que realizan acciones de bien público, asistiéndolas en el desarrollo de planes de comunicación y relaciones públicas.

Vincular a los estudiantes del Brand PR Máster Universitario en Marketing y Comunicación Corporativa con la realidad y complejidad del campo profesional y los expertos que en él se desempeñan.

Articular el programa de Máster y sus actividades con las distintas áreas y departamentos de la Universidad. Sensibilizar a la comunidad universitaria en su conjunto de su función, compromiso y responsabilidad social que le concierne.

Visibilizar las actividades y acciones formativas que lleva adelante la Universidad.

Uno de los propósitos de la actividad fue articular el programa de Máster y sus actividades con las distintas áreas y departamentos de la Universidad. El personal implicado y sus funciones, los recursos y la infraestructura utilizada fueron:

Equipo de Máster (Director y Coordinadoras): responsables del diseño de la idea, planificación su implementación y evaluación.

Profesorado de Máster: formación en marketing social de los estudiantes consultores.

Consultores: son estudiantes del Brand PR Máster Universitario en Marketing y Comunicación Corporativa de la Universidad San Jorge. Cada ONG seleccionada dispondrá de un consultor cómo mínimo. Su función principal es acompañar a la ONG en todo el proceso. Para ello, deberá tener un primer contacto -presencial o telefónicamente-, previo a la sesión, con la organización asignada y ayudarle a elaborar un resumen (brief) sobre ella. Dicho resumen deberá ser entregado antes del día del maratón a los profesionales asesores (dos asesores mas un líder) y expuesto conjuntamente (consultores y representantes de las ONG's) en la sesión ante esos mismos profesionales asesores. Los consultores deberán encontrarse con los participantes 15 minutos antes del comienzo de su horario para instruirlos y acompañarlos a participar en la actividad. Cada ONG dispone de dos horas para resolver junto a los

3 [Disponible en http://www.dircom.org/Actualidad-Dircom/socios-de-dircom-aragon-participan-en-el-maraton-solidario-en-comunicacion-corporativa-de-la-usj.html] Consultado el 10 de junio de 2013. 
profesionales asesores el problema específico de comunicación, que se ha detectado previamente.

Representantes de la ONG: son los miembros de las ONG seleccionadas para el Maratón, quienes tienen la responsabilidad de brindar información veraz tanto a los consultores como a los profesionales a lo largo de todo el proceso. Cada ONG's debe estar representada por un mínimo 3 integrantes de la entidad. Deberán preparar un resumen, con la ayuda de los consultores, de su propia organización y exponerlo en la reunión -detallada al inicio- con los profesionales asesores.

Profesionales asesores: especialistas en Marketing y Comunicación Corporativa que analizan la situación de la ONG y le brindan consejos útiles para una planificación integral de la comunicación. Participan consultores, Dircoms, docentes, periodistas, publicistas, investigadores, escritores, referentes sociales y especialistas en las temáticas de algunas ONG's, como en salud. Esta interdisciplinariedad otorga mayor riqueza a la actividad.

Líder: forma parte del grupo de profesionales asesores y es su figura principal. Es el encargado de guiar y coordinar la reunión. Debe ser un experto en pensamiento creador. Su función es formular claramente el problema y que todos se familiaricen con él. Cuando lo haga, debe estimular ideas, hacer que se rompa el hielo en el grupo. Es el encargado de que se cumplan las normas, no permitiendo las críticas. Debe permanecer callado e intervenir cuando se corte la afluencia de ideas. Su función también es la de incentivar a los implicados para que todos participen y den ideas, conceder la palabra. Si el brainstorming se estanca, es quien debe ayudar a avivar el fuego. Está comprobado que los estímulos sensoriales (luces, sonidos, gustos dulces, etc.) favorecen la producción de ideas, por lo que se recomienda utilizar los recursos disponibles en la sala de trabajo.

Representantes del Consejo Profesional de Relaciones Públicas de Argentina: responsables de la inducción inicial para la organización, la capacitación de los consultores y la supervisión del desarrollo de la misma.

Información Universitaria Posgrados: difusión de la actividad entre los DIRCOM de Zaragoza, convocatoria a profesionales asesores.

Unidad de Orientación Profesional y Empleo: difusión de la actividad entre los DIRCOM de Zaragoza, convocatoria a profesionales asesores. Brinda asesoramiento sobre posibilidades de inserción laboral y profesional en la Organizaciones interesadas en continuar vinculadas a la USJ.

Instituto Humanismo y Sociedad: difusión entre las ONG's. Elaboración del informe para la selección. Ofrecimiento de la posibilidad de entablar convenidos de colaboración con las ONG's participantes

Profesores Facultad de Comunicación: convocados a participación y colaboración como profesionales asesores. 
Entidades Colaboradoras: difusión de la actividad, convocatoria a profesionales asesores y participación en la conferencia final.

\subsection{La experiencia}

Para el cumplimiento de los objetivos, el desarrollo de la práctica implicó diversas fases y etapas. Estas etapas tuvieron especial desarrollo, particularmente en la primera edición (2012), replicándose en el año 2013, aquellas que permitieron llevara adelante la organización de la actividad.

a) Preparación. Comprendió el estudio de la iniciativa llevada a cabo en Argentina (contacto con los responsables, lectura de fichas y material de soporte) y asesoramiento sobre los modos de implementar la misma en España. Luego se diseño una propuesta inicial para ser llevada a cabo en la Universidad, previa verificación y autorización por parte del Decanato de la Facultad de Comunicación y Vicerrectorado de Ordenación Académica y Unidad Técnica Calidad; y las correspondientes consultas al Departamento de Información Universitaria de Posgrado y el Instituto de Humanismo y Sociedad. Finalmente se realizaron contactos y reuniones con socios y colaboradores para presentar la propuesta: Consejo Profesional de Relaciones Públicas de la Argentina, Caritas Diocesana Zaragoza y DIRCOM Aragón.

b) Convocatoria. Incluyo: la difusión de la actividad a nivel interno en la Universidad y a la sociedad en general; la convocatoria a través del Instituto de Humanismo y Sociedad, a las ONG's para participar, las cuales deben cumplimentar una ficha de inscripción; la recepción de las fichas de inscripción de las ONG's; y la selecciona las propuestas en base a criterios definidos por expertos, elaborando un informe detallado.

Además, la Dirección del Máster realizó la convocatoria a los estudiantes del programa para ser consultores, a través de una charla introductoria de la actividad y mediante la Plataforma Docente Universitaria (Campus virtual). También convocó a los profesionales asesores (Directores de Comunicación y Profesores de la USJ) con el aporte del Departamento de Información Universitaria de Posgrado y la Unidad de Orientación Profesional y Empleo, y realizó la convocatoria abierta a la conferencia de cierre del maratón y difusión en medios.

c) Trabajos previos. Tuvo que ver con lo relativo al:

Envío de documentos en los que se detallan las tareas de consultores, asesores y coordinadores; capacitación a los consultores vía teleconferencia a cargo de un responsable del Consejo Profesional de Relaciones Públicas de la República Argentina;

Desarrollo de la clase de Marketing Social del programa de Máster en la que se capacita a los consultores en habilidades específicas a ser empleadas durante el Maratón. 
Asignación de consultores para las entidades seleccionadas. El consultor estudia la ficha cumplimentada, toma contacto con los representantes de la ONG para intercambiar información y elaborar un diagnóstico de comunicación enfocado a detectar problemas. El contacto fue presencial o telefónico.

Definición, confirmación y coordinación de la agenda de cada sesión del Maratón, estableciendo qué profesionales trabajarán juntos asesorando a cada organización.

Los días previos se llevó adelante la preparación de programas, salas, espacios de café, material de trabajo, etc.

d) Celebración.

- Durante el Maratón:

El día del Maratón se asignó una sesión de dos horas a cada ONG. Durante la misma, los profesionales brindaron su asesoramiento y los consultores tomaron nota de las recomendaciones.

Previo a la sesión, los consultores recibieron a los representantes de las ONG's en un espacio de café en donde la Dirección del Máster los presentó al panel de expertos profesionales convocados.

Como actividad de cierre se ofreció una charla para capacitar a aquellas ONG's que no ingresaron al formato tradicional de asesoramiento. La misma fue brindada por especialistas en comunicación para el Tercer Sector.

- Durante las sesiones:

Presentación de la entidad a cargo de los representantes (entre 10 y 15 minutos máximo):

Objetivo: Ej. La lucha contra el cáncer.

Características: Ej. Somos 40 personas, 30 voluntarios y 10 rentados, tenemos sedes en todo el país...

Breve historia: Ej. ...fue creada por Dr. XX en el año XX...

Material gráfico: si la entidad dispone de folletos, posters, brochure institucional), es recomendable que se exponga en la reunión.

Planteamiento del problema a resolver (10 minutos): Los consultores, junto con los tres representantes de la entidad participante deben explicar cual es la situación a mejorar. Debe recordarse que, si bien la mayoría de las ONG's requieren de financiamiento y es posible que la comunicación fundraising sea un tema importante para ellas, ésta no es una reunión de recaudación de fondos. No obstante, si lo que se pretende es dedicar la sesión a tratar de ver como mejorar su comunicación con sponsors debe quedar claro que el planteamiento es desde la comunicación. Por lo tanto, algunos de los posibles problemas son:

No tenemos un plan de comunicación y quisiéramos ideas para elaborarlo. 
Tenemos un problema específico (Ej. Nuestro objeto es la lucha contra la poliomielitis y ésta se erradicó)

Quisiéramos una mejor comunicación interna (o con donantes, o con la prensa, etc.)

Necesitamos de una ley y no sabemos como empezar.

Una vez planteado el problema, los participantes realizan un brainstorming o lluvia de ideas que estará dirigido por el Líder. El rol del consultor no es el de participar en el brainstorming (se amplia información al respecto, más adelante) sino el de relator de lo sucedido. Para ello fue necesario que los consultores tomasen nota de las ideas planteadas para luego, finalizada la reunión, efectuar una devolución a la ONG. La lluvia de ideas se desarrollará durante aproximadamente una hora. Los 20 minutos siguientes se dedicarón a efectuar un resumen común que el consultor deberá documentar minuciosamente (habitualmente será un plan de trabajo para los próximos meses, o la reformulación de la visión y objetivos de la ONG, o el desarrollo de una idea (Ej. Un evento o una campaña de prensa). La tarea, por lo tanto, consistió en descubrir soluciones:

Bajo su dirección se elabora una lista definitiva de ideas, para seleccionar las que parecen más interesantes.

La selección se realiza desechando las ideas que no tienen valor y se estudia si son válidas las que se consideran interesantes. Lo mejor es establecer una lista de criterios de conveniencia o no de cada idea.

Se seleccionan las ideas más útiles y si es necesario se ponderarán. Pueden realizarlo los mismos miembros del grupo o crear otros para esta tarea, clasificadas por categorías (tarea que corresponde al líder).

Los 20 minutos siguientes se dedicarán a efectuar un resumen común que deberá documentar minuciosamente (habitualmente será un plan de trabajo para los próximos meses, o la reformulación de la visión y objetivos de la ONG's, o el desarrollo de una idea (Ej. un evento o una campaña de prensa).

Los 10 minutos finales se dedicarán a realizar una puesta en común. El consultor o consultores, se encargarán de documentar el trabajo de la reunión. Tras la correspondiente presentación, los profesionales asesores expondrán sus recomendaciones. Serán los consultores quienes deban tomar nota de dichas sugerencias, aportando, si lo consideran oportuno, su punto de vista.

e) Post evento. Finalizado el Maratón, los consultores elaboró el informe que, antes de ser entregado a la ONG en el lapso de los 30 días posteriores a la sesión, fue revisado y corregido por los profesionales asesores. Quedó a criterio de los consultores dar seguimiento al trabajo de la ONG luego de la devolución. A su vez, la Unidad de Orientación Profesional y Empleo (UOPyE) brindó asesoramiento sobre posibilidades de inserción laboral y profesional en la Organizaciones interesadas en continuar 
vinculadas a la USJ. Por su parte, El Instituto de Humanismo y Sociedad ofreció la posibilidad de entablar convenidos de colaboración con las ONG's participantes.

Además se procedió a la evaluación de las actividades por parte de los estudiantes consultores en una nueva sesión de la asignatura Marketing Social. También se abrió un espacio en la Plataforma Docente Universitaria para que los estudiantes formulasen libremente opiniones y comentarios sobre la experiencia. Se enviaron saludos de agradecimiento a todos los participantes y colaboradores de la experiencia.

\section{Discusiones y conclusiones}

Para la evaluación de la práctica por parte de los estudiantes se empleó un Informe de trabajo de campo, en el que los estudiantes, dentro del grupo de trabajo, puedan demostrar ser capaces de:

realizar un diagnóstico preciso del problema presentado por la entidad

desarrollar un análisis argumentado y apoyado en el asesoramiento de expertos y fuentes bibliográficas

diseñar un plan de acción con objetivos concretos.

Estos criterios de evaluación fueron incorporados a la asignatura Marketing Social. Con este estrategia, la principal herramienta de aprendizaje para los estudiantes lo constituye el rol play que los mismos deben desarrollar durante las sesiones. $\mathrm{Su}$ función principal es acompañar a la ONG en todo el proceso de diagnóstico y planificación de las soluciones necesarias.

A su vez los estudiantes, previo a la experiencia, se han capacitado y formado en habilidades específicas a ser empleadas durante el Maratón, en la asignatura Marketing Social del programa de Máster.

La práctica de aprendizaje servicio permite a los estudiantes vincular la realidad a la que deberán enfrentarse como profesionales, con la experiencia de quienes ya se desenvuelven en dicho campo, poniendo de manifiesto las competencias y habilidades adquiridas; sumando el aporte solidario de la acción de responsabilidad social que se lleva adelante. La realización del Maratón nos ha permitido obtener resultados en función de los objetivos propuestos, que a continuación mencionamos agrupados según los tipos actores: los estudiantes, el programa de Máster y su profesorado; y el contexto.

En los estudiantes, la práctica de esta actividad ha sido muy positiva para la aplicación y puesta en práctica de las competencias y habilidades del Programa de Máster. Los estudiantes han podido conocer de primera mano el "panorama" de la realidad profesional en su campo de actuación. Esto les ha permitido descubrir la problemática comunicativa de un colectivo que normalmente, por falta de medios, recursos humanos, o conciencia del aporte que la gestión de comunicación posibilita, no dispone de 
las condiciones óptimas para llevar a cabo acciones en este campo. Los estudiantes han aprendido de manera empírica a enfrentarse a situaciones reales que luego en el propio ejercicio profesional serán cotidianas.

En el Programa de Máster y su profesorado, el impacto de esta práctica en el programa se ha dado en dos sentidos: la difusión que ha tenido la actividad en particular ha contribuido a que el Programa de Máster y la Universidad acrecentaran su notoriedad con más profundidad en sectores de la población de Zaragoza, España y a nivel internacional, que o bien ya los conocían pero no "tenían en consideración", o que desconocían por completo. Para el profesorado implicado, a demás de ser una experiencia personal y profesional muy enriquecedora, ha podido comprobar el grado de compromiso y conocimiento de los estudiantes a los que les han transmitidos dichos enseñanzas. A su vez, el programa se ha enriquecido notablemente con prácticas docentes innovadoras que nutren y mejoran las dinámicas y estrategias didácticas que ya se vienen empleando. Al mismo tiempo se han fortalecido los vínculos con sectores profesionales de la comunicación que nos permitirán en un futuro una mayor excelencia en el claustro de profesores y reforzar la oferta de entidades en las que se desarrolla el Módulo de Prácticas Externas.

En el contexto (resto de la Universidad, ONG's, profesionales de la comunicación y el marketing, entidades colaboradoras), tanto para la Universidad en general como para el resto de la sociedad aragonesa (incluso el resto de la sociedad nacional puesto que la celebración del Maratón se ha conocido en distintos puntos de España-) ha supuesto una reflexión profunda sobre la responsabilidad social que le cabe tanto a la Universidad (en su conjunto de colectivos que representa) como a los Profesionales del Marketing y la Comunicación Corporativa. Para las ONG's y sus integrantes han podido observar y reflexionar sobre los problemas de comunicación que conllevan un bajo conocimiento de su labor social, por parte de su entorno más próximo y los ciudadanos a los que se dirigen; y las potencialidades que tiene incorporar a sus equipos profesionales como los que forma el propio Máster.

Si bien la iniciativa ya tiene antecedentes en Argentina, nunca se había implementado con estudiantes de Máster, ni tampoco en España. La práctica, entre sus aspectos innovadores, combina en una misma acción los siguientes distintos componentes:

Solidario: motiva la responsabilidad social y participación en acciones de voluntariado

Formativo: los estudiantes consultores completan las competencias y conocimientos adquiridos en el marco del programa de Máster.

De inserción laboral: se sensibiliza a las entidades sobre el aporte que tiene la comunicación a sus actividades y a los estudiantes se les muestra posibilidades laborales reales.

De desarrollo del campo profesional: se amplia al tercer sector los ámbitos de desempeño profesional para los que forma el programa. 
De vinculación institucional: con organizaciones del sector con las que no se tenía relación desde el Programa de Máster como DIRCOM y AMAPA (Asociación de Medios y Agencias de Publicidad de Aragón) y con la comunidad en general.

De difusión: el alto impacto innovador y creativo de la actividad, la acogida entre los participantes y los excelentes resultados se reflejan en las repercusiones mediáticas obtenidas, y en las distintas menciones y agradecimientos brindados por las Instituciones participantes.

La práctica aquí desarrollada se trata de una experiencia sin ánimo de lucro en la que se pretende poner en contacto conocimientos, experiencias y personas de distintos ámbitos que pueden complementarse. En este caso profesionales y docentes de la comunicación y el marketing con trayectorias importantes; estudiantes con ganas de aportar con esfuerzo, compromiso y energía, sus conocimientos, habilidades y competencias adquiridas; $y$ personas involucradas voluntaria o profesionalmente con el desarrollo de la sociedad civil. Así, el Maratón Solidario de Comunicación Corporativa, es perfectamente un modelo de innovación docente replicable y transferible en otros contextos didácticos y desde luego extrapolable a cualquier otra realidad social.

\section{Referencias bibliográficas}

ARAÚJO, Ulises y ARANTES, Valeria (2010): Aprendizaje Basado en Problemas y construcción de Ciudadanía: el proyecto de la Universidad de Sao Pablo, en MARTÍNEZ Miquel (ed.): Aprendizaje servicios y responsabilidad social de las Universidades. Octaedro-ICE. Barcelona.

CECCHI, Néstor Horacio (2006): Aprendizaje Servicio en Educación Superior. La experiencia latinoamericana, en Seminario Internacional Responsabilidad Social Universitaria: Aprendizaje Servicio, Universidad Central de Venezuela. Disponible en http://www.documentacion.edex.es/docs/0406CECapr.pdf. Consultada el 20 de septiembre de 2013.

DAYNES, Gary y LONGO, Nicholas (2004): Jane Addams and the Origins of Service-Learning Practice in the United States, en Michigan Journal of Community Service Learning. Disponible en: http:/quod.lib.umich.edu/m/ mjcs1/3239521.0011.101 ?rgn=main;view=fulltext. Consultada el 20 de septiembre de 2013.

FOLGUEIRAS, Pilar, LUNA, Esther y PUIG, Gemma (2013): Aprendizaje y servicio: estudio del grado de satisfacción de estudiantes universitarios" en Revista de Educación. Disponible en: http://www.revistaeducacion.mec.es/doi/362_157.pdf. C Consultada el 20 de septiembre de 2013.

FRANCISCO, Andrea y MOLINER, Lidón (2010): El Aprendizaje Servicio en la Universidad: una estrategia en la formación de ciudadanía crítica, en Revista Electrónica Interuniversitaria de Formación del Profesorado. Disponible en: http:// www.aufop.com /aufop/uploaded_files/articulos/1291992629.pdf. Consultada el 20 de septiembre de 2013. 
MARTA LAZO, Carmen y ALDEA, Patricia (2012): El aprendizaje-servicio, una herramienta para el desarrollo profesional de la responsabilidad social del periodista, en Estudios sobre el mensaje periodístico, (18), 577-588.

MARTÍNEZ-ODRÍA, Arantzazu (2007): Service Learning o Aprendizaje-Servicio. $\mathrm{La}$ apertura de la escuela a la comunidad local como propuesta de educación para la ciudadanía", en Bordón. Disponible en: www.dialnet.unirioja.es/servlet/ fichero_articulo?codigo =2582784. Consultado el 18 de septiembre del 2013.

MARTÍNEZ, M. (2010): Aprendizaje Servicio y construcción de ciudadanía Activa de la Universidad: la dimensión social y cívica de los aprendizajes académicos, en MARTÍNEZ Miquel (ed.): Aprendizaje servicios y responsabilidad social de las Universidades. Octaedro-ICE. Barcelona.

MARTÍNEZ, M., FRANCISCO, E. (2005): Una propuesta de formación ciudadana para las EEES, en Revista Española de pedagogía, 230, 63-84.

MARTÍNEZ, Miquel (ed) (2010): Aprendizaje servicio y responsabilidad social de las Universidades. ICE y Ediciones Octaedro. Barcelona.

NAVAL, C. (2010): Universidad y conciencia cívica. Algunas experiencias fructíferas: Service learning y Campus Compact, en MARTÍNEZ Miquel (ed.): Aprendizaje servicios y responsabilidad social de las Universidades. Octaedro-ICE. Barcelona.

PUIG ROVIRA, José María y PALOS RODRÍGUEZ, J.M. (2006): Rasgos pedagógicos del aprendizaje servicio, en Cuadernos de Pedagogía, 357, 60-63.

PUIG, José María (coord.) (2009): Aprendizaje Servicio. Educación y compromiso cívico. Graó. Barcelona.

\section{Los autores}

Alejandro Álvarez Nobell. Doctor en Dirección Estratégica de la Comunicación y Máster en Gestión Estratégica e Innovación en Comunicación (Málaga, España). Lic. en Comunicación Social. (Córdoba, Argentina). Actualmente es Secretario de Ciencia y Tecnología en la Escuela de Ciencias de la Información (Universidad Nacional de Córdoba, Argentina). Investigador y Docente de Grado y Posgrado. Autor de los Libros "Medición y Evaluación en Comunicación" (2011) y "Brand PR: Las Relaciones públicas de Marca" (2013); además de diversos capítulos y artículos en revistas científicas. Entre el 2011 y 2013 fue Director del BrandPR Máster en Marketing y Comunicación Corporativa y profesor en la Facultad de Comunicación de la Universidad San Jorge (España). Integrante de redes académicas y profesionales en comunicación (RAIC, SLCS, AE-IC; FISEC, CPRP, IIRP) y diversos Comités Científicos, Académicos y en Congresos, Publicaciones y Actividades Académicas. Consultor en Comunicación, Imagen e Identidad Mediática.

Nerea Vadillo Bengoa. Licenciada en Periodismo y Doctora en Ciencias Políticas y Sociología por la Universidad del País Vasco. Master en Integración Europea. Dircom de la Facultad de Comunicación de la Universidad San Jorge, de la que fue su primera 
decana (2008-2012). Profesora de grado y posgrado en Periodismo. Ha sido profesora-investigadora en el departamento de Derecho Internacional Público y Relaciones Internacionales de la Universidad del País Vasco y de la Universidad Complutense de Madrid. Ha impartido docencia en la University of California y ha sido Visiting Research Fellow en la School of International Studies de la University of Miami y en el Instituto Superior de Relaciones Internacionales "Raúl Roa" (ISRI) de La Habana (Cuba). Investigadora Principal del Grupo de Educomunicación (GIEC), grupo emergente del Gobierno de Aragón. Autora del libro "La Política Exterior de la Unión Europea hacia Cuba 1993-2003" y es además autora de diversos artículos de revistas científicas de ámbito nacional e internacional y de diferentes capítulos de libro. 